\title{
CARCINOMA PÓS-TRANSPLANTE HEPÁTICO
}

\author{
CARCINOMA AFTER LIVER TRANSPLANTATION
}

\author{
Júlio Cezar Uili Coelho, TCBC-PR ${ }^{1}$ \\ Mauro Rafael da Igreja ${ }^{2}$ \\ Mônica Beatriz Parolin ${ }^{3}$
}

\section{INTRODUÇÃO}

Desde o início da década de 60 mais de 400.000 transplantes foram realizados no mundo ${ }^{1}$. Os pacientes receptores de órgãos sólidos são mantidos sob terapia imunossupressora por períodos prolongados, tornando-se suscetíveis ao desenvolvimento de uma variedade de neoplasias. Com o aumento na sobrevida pós-transplante e a crescente aceitação de candidatos de faixa etária mais avançada é inevitável que maior número de receptores de órgãos possam desenvolver neoplasias. O objetivo do presente artigo é relatar o caso de um paciente com carcinoma epidermóide de faringe detectado dois anos após o transplante hepático.

\section{RELATO DO CASO}

Paciente com 42 anos, do sexo masculino, branco, portador de cirrose hepática alcóolica descompensada e em abstinência há dois anos, foi submetido a transplante ortotópico de fígado em novembro de 1996 no nosso serviço. O paciente era tabagista crônico há 20 anos (20 cigarros/dia). Durante a avaliação pré-transplante nenhuma lesão em palato foi identificada.

O paciente desenvolveu como complicação pós-transplante, trombose da artéria hepática, tendo sido submetido a retransplante em junho de 1997, evoluindo sem intercorrências. O esquema de imunossupressão utilizado nos dois transplantes foi a associação de ciclosporina, prednisona e azatioprina. Apesar de orientado, o paciente persistiu com o tabagismo durante todo o período pós-transplante. Durante consulta médica de rotina em janeiro de 1999, queixou-se de obstrução nasal persistente à direita e sensação de corpo estranho na faringe. Avaliado pelo Serviço de Otorrinolaringologia diagnosticou-se lesão nodular esbranquiçada e elevada de $0,5 \mathrm{~cm}$ de diâmetro, localizada em palato à direita da linha média, e ausência de linfadenomegalia. A lesão foi biopsiada e o exame anatomopatológico revelou carcinoma epidermóide moderadamente diferenciado invasor. A fibrobroncoscopia e a endoscopia digestiva alta foram normais. $\mathrm{O}$ tratamento realizado foi radioterapia radical com acelerador linear, exclusiva de palato, e drenagem linfática regional, não sendo modificado o esquema de imunossupressão. A dose inicial de $5.000 \mathrm{cGy}$ em 25 sessões foi seguida de uma dose complementar de $2.000 \mathrm{cGy}$. Houve remissão completa da lesão e, após 12 meses, o paciente continua assintomático e sem recidiva da lesão.

\section{DISCUSSÃO}

Os pacientes submetidos a transplantes têm incidência aumentada de tumores, tanto tumores "de novo", como recidivas ou transmissões inadvertidas de tumor com o enxerto. Estas neoplasias são diferentes daquelas da população geral e sua incidência varia conforme o tipo de órgão transplantado, esquema de imunossupressão e tempo de acompanhamento. Duas séries recentes que analisaram transplantados renais acompanhados por 20 anos identificaram $40 \%$ e $50 \%$ de tumores, comparados com $6 \%$ em um grupo com idade semelhante à da população geral. ${ }^{2} \mathrm{Os}$ dados do Centro de Registro de Tumores Cincinatti (CRTC), que tem mais de 8.000 tumores pós-transplante cadastrados, mostram que as neoplasias comuns na população (câncer de pulmão, mama, tumor invasivo de colo de útero) não têm incidência aumentada nesses pacientes. Entretanto, existe acentuado aumento de certos tumores, como de pele e lábios (10 a 29 vezes), linfomas não-Hodgkin ou doença linfoproliferativa pós-transplante ( 28 a 49 vezes), sarcoma de Kaposi (400 a 500 vezes), neoplasias de ânus, períneo e genital (dez vezes), carcinoma hepatocelular (20 a 38 vezes) e carcinomas renais ${ }^{3,5}$.

1. Professor Titular e Chefe dos Serviços de Cirurgia do Aparelho Digestivo e de Transplante Hepático da UFPR.

2. Residente dos Serviços de Cirurgia do Aparelho Digestivo e de Transplante Hepático da UFPR.

3. Médica do Serviço de Transplante Hepático da UFPR.

Recebido em 27/3/2000

Aceito para publicação em 18/12/2001

Trabalho realizado no Serviço de Transplante Hepático da Universidade Federal do Paraná — UFPR. 
O carcinoma epidermóide de faringe, objeto do presente relato, não é um tipo de tumor freqüentemente descrito no período pós-transplante. Por tratar-se de paciente com exposição crônica a carcinógenos conhecidos, tais como tabaco e álcool, é provável que a imunossupressão tenha apenas facilitado o aparecimento da lesão. Dos tumores registrados no CRTC, foram relatados apenas casos de tumores de faringe tratados previamente ao transplante e que apresentaram um alto índice de recidiva pós-transplante $(60 \%)^{1,5}$. Parece que a terapia de imunossupressão limita a capacidade do sistema imunológico de eliminar células neoplásicas preexistentes ou induzidas por vários carcinógenos.
No tratamento desses tumores parece lógico que uma redução da imunossupressão permitiria a recuperação do sistema imunológico e o controle da doença. Existe algum benefício apenas nas neoplasias relacionadas a oncovírus e particularmente nos linfomas (19\% de remissão completa do tumor com a redução) e no sarcoma de Kaposi (39\% de remissão completa). Os outros tumores, especialmente os epiteliais, não respondem a uma redução da imunossupressão, o que justifica nossa decisão de não alterar o regime imunossupressor do caso relatado. Além disso, a redução traz um alto risco de rejeição e perda do enxerto.

\begin{abstract}
The number of organ transplantation has increasing worldwide. Several authors have reported an increase in cancer incidence in these patients. There is a marked increase of a variety of tumors. However, common cancers seen in the general population showed no increase. The authors describe a case of a 42-year-old male with alcoholic cirrhosis who underwent orthotopic liver transplantation. The patient developed an uncommon solid tumor two years post transplantation, an epidermoid carcinoma of the pharynx. Radical radiotherapy of the palate was performed and no change was made in immunosuppression therapy. Ten months later the patient is doing well with no evidence of local recurrence or metastatic disease.
\end{abstract}

Key Words: Neoplasms; Organ transplantation; Immunossuppression.

\title{
REFERÊNCIAS
}

1. Penn I. The effect of immunosuppression on pre-existing cancers. Transplantation, 1993, 55:742-747.

2. Gazdar F. Tumor arising after organ transplantation - Sorting out their origins. JAMA, 1997, 227:154-155.

3. Penn I. Post transplant malignancies. Transplant Proc, 1999, 31:1260-1262.

4. Penn I. Evaluation of the candidate with a previous malignancy. Liver Transplant Surg, 1996, 2(Suppl 1):109. 113.
5. Penn I. Neoplasia: An example of plasticity of immune response. Transplant Proc,1996, 28:2089-2093.

Endereço para correspondência:

Dr. Júlio Cezar U. Coelho

Rua Bento Viana, 1140/2202

80240-110 - Curitiba-PR

Tel./Fax.: (41) 322-3789 